\title{
RENORMINGS OF VASAK SPACES
}

\author{
Gaj Ram Damai ${ }^{1}$, Prakash Muni Bajracharya ${ }^{2}$, Yongjin Li $^{3} \S$ \\ ${ }^{1}$ Siddhnath Science Campus (T.U.) \\ Mahendranagar, NEPAL \\ ${ }^{2}$ Central Department of Mathematics (T.U.) \\ Kirtipur, Kathmandu, NEPAL \\ ${ }^{3}$ Department of Mathematics \\ Sun Yat-Sen University \\ Guangzhou, 510275, P.R. CHINA
}

\begin{abstract}
In this paper, we present the basic facts about vasak (WCD) space with application to the construction of equivalent norm on the vasak spaces. We study nearly about an open problem raised in [1].
\end{abstract}

AMS Subject Classification: 46B22, 46B20

Key Words: Asplund spaces, LUR norm, WCGs

\section{Introduction}

A fundamental concept in non separable Banach space theory is the concept of weakly compactly generated spaces, introduced and studied first by Corson and Lindenstrauss (cf. [15] and references therein). This concept allows for splitting many non separable Banach spaces into separable ranges of projections and generates nice geometric and topological properties of such spaces. A class of spaces wider than weakly compactly generated space (WCG), known as weakly countably determined (WCD) or Vasak space. It was originally defined and investigated by Vasak [18]. So it necessary to get the relation between them.

Received: $\quad$ September 1, 2016

Revised: $\quad$ October 9, 2016

Published: December 2, 2016

$\S_{\text {Correspondence author }}$ (c) 2016 Academic Publications, Ltd. url: www.acadpubl.eu 
The WCG property has been an active topic of research for several years, generalization of this property has been investigated. Vasak in his famous paper [4] defined a more general class of the Banach spaces than WCG spaces are: He says that a Banach space $X$ is WCD space, now also known as Vasak space if, there is a subspace $\sum^{\prime}$ of Baire's space $\sum=\mathbb{N}^{N}$ where $\mathbb{N}$ is the nature numbers with discrete topology, $\mathbb{N}^{N}$ is set of natural numbers with product topology, and an upper semi-continuous, multi valued valued and surjective mapping $\psi$ from $\sum^{\prime}$ onto $X$ such that $\psi(\sigma)$ is non empty compact set for all $\sigma \in \sum^{\prime}$ and that the set $\left\{\sigma \in \sum^{\prime}: \psi(\sigma) \bigcap C \neq \phi\right\}$ is closed for all weakly closed subset $C$ in $X$. In fact, Vasak dealt with PRI for WCD spaces.

In his paper, Vasak also showed by adapting the method used for WCG space, Vasak space is renormable in the LUR manner. Recently Mercourakis [9] has shown that, if $\mathrm{X}$ is a Vasak Space then $X^{*}$ is dually strictly convexifiable. Many of the renorming results for WCG spaces are valid for Vasak spaces, so we push these results ahead in this direction. The important advantage of WCD Banach space over WCG spaces are that they are stable with respect to subspaces [1].

Let $X$ be a Banach space whose dual $X^{*}$ is a WCD, then an equivalent LUR norm on $X$ is constructed. According to a recent example of Mercourakis, this is a real extension of earlier result of Godefroy, Troyanski, White-field, and Zizler obtained in [3]. We obtain a new construction of a LUR norm on a Vasak space and further cultivation of this method yields the new result that every dual Vasak space admits a dual LUR norm [6]. The WCG property is generalized without losing the most useful properties of it is studied in [18]. The deep result of $\mathrm{D}$. Amir and $\mathrm{J}$. Lindenstrauss form the basis of our discussion .The renorming theorem of Troyanski is derived and some of its ramifications are discussed .A number of basic technical results are developed for the study of the structure of WCD space [4]. We investigate how vasak spaces are renormed by WLUR and Frechet differentiable norm

\section{Notations and Terminologies}

In the sequel, $(X,\|\cdot\|)$ is the real Banach space with norm $\|\cdot\| ; S(X)$ is the unit sphere in $X ;\left(X^{*},\|\cdot\|^{*}\right)$ is the dual space of $X ; S\left(X^{*}\right)$ is the unit sphere of $X^{*} ; B(X)$ is the unit ball of $X ; B\left(X^{*}\right)$ is the unit ball of $X^{*} ; X^{* *}$ is the second dual of $X$; WCG, WCD, LUR, PRI are short form of weakly compactly generated, weakly countably determined(vasak Banach space), locally uniform rotund, projection resolution of identity respectively. 


\section{Definitions}

2.1. A Banach space $X$ is called Vasak space, if there is a countable collection $\left\{B_{n}\right\}$ of $w^{*}$-compact subsets of $X^{* *}$ such that for all $x \in X$ and $u \in X^{* *} \backslash X$ there exists $n_{0} \in \mathbb{N}$ for which $x \in B_{n_{0}}$ and $u \notin B_{n_{0}}$.

Equivalently, $\mathcal{B}$ be the countable family of subsets of $w^{*}$ compact subsets of $X^{* *}$ such that for all and $u \in X^{* *} \backslash X$ there is $B \subseteq \mathcal{B}$ such that $x \in B$ and $u \notin B$.

2.2. [18] Let $T^{\prime}$ be a topological space, $T \subseteq T^{\prime}$. We say that $T$ is countably determined (CD) if and only if there are countable compacts $\left\{A_{i}: i \in \omega\right\} \subseteq T$ such that for all $x \in T$ there is $\rho \subseteq \omega$ such that $x \in \bigcap\left\{A_{i}: i \in \rho\right\} \subseteq T$. Thus we say that $T$ is determined by $\left\{A_{i}: i \in \omega\right\}$ in $T^{\prime}$ where $\omega$ denotes the set of all natural numbers and the same time first infinite ordinal number.

2.3. A norm $\|\cdot\|$ of a Banach space $X$ is called 2-rotund (2R) if $\left\{f_{n}\right\}$ is norm convergent whenever $x_{n} \in S(X)$ are such that $\lim _{m, n \rightarrow \infty}\left\|x_{m}+x_{n}\right\|=2[11]$.

2.4. If $M$ is a bounded total set in $X$, we will say that the norm $\|\cdot\|$ on $X$ is dually $M$-2-rotund (M-2R) if $\left\{f_{n}\right\}$ is convergent to some $f \in B\left(X^{*}\right)$ uniformly on $M$ whenever $f_{n} \in S\left(X^{*}\right)$ are such that $\lim _{m, n \rightarrow \infty}\left\|f_{m}+f_{n}\right\|=2$.

2.5. A Banach space $X$ is said to be WCG if it contains weakly compact total set [11].

We frequently work on the class of Vasak spaces which contains all separable, reflexive spaces and provide a most appropriate setting for many questions on non separable Banach spaces. The sets in the definition of Vasak spaces can be taken convex and symmetric that every subspace of a WCG space is a Vasak space. Recall that if $X$ is WCG space then for such sets we may take a reindex family of $n B_{X}+\frac{1}{m} K, m, n \in \mathbb{N}$, where $K$ is weakly compact set that generates $X$.

Examples. WCG, separable, reflexive, and $C_{0}(\Gamma)$ spaces for some $\Gamma$ spaces are WCD spaces.

We have the following problem:

If a Banach space $X$ is Vasak space, does $X$ admit a norm with the following property: if a sequence $\left\{f_{n}\right\}$ is weak*-convergent to some $f \in B\left(X^{*}\right)$ whenever $S\left(X^{*}\right)$ are such that $\lim _{m, n \rightarrow \infty}\left\|f_{m}+f_{n}\right\|=2[1]$. 


\section{Theorems}

We consider more general situations when $X$ is a Vasak space.

Theorem 3.1. If $X^{*}$ is Vasak, then $X$ admits an equivalent Frechet differentiable norm.

To prove this theorem, we require the following technical lemmas.

Lemma 3.2. ([3], T 2.3, pp. 290). If $X$ be a Banach space, then

(1) If $X$ is WCD Banach space, then $X$ admits LUR norm.

(2) If $X$ is a dual WCD Banach space, then $X$ admits dual LUR norm.

Lemma 3.3. ([3], Cor. 1.17, $p$ 289) If $X$ is a Banach space such that $X^{*}$ is WCD, then $X$ admits a WLUR norm $\|\cdot\|$.

Lemma 3.4. ([3], p. 296) Let $(X,\|\cdot\|)$ be a Banach space and if the norm is WLUR and the norm $\|\cdot\|$ is Frechet differentiable, then $X$ admits LUR norm.

Thus using these lemmas by means of transfer and Asplund averaging techniques we prove the theorem as follows:

Proof of Theorem 3.1. By lemma 3.2, $X$ admits a norm $\|\cdot\|_{1}$ whose dual norm is LUR norm and by lemma $3.3, X$ admits a WLUR $\|\cdot\|_{2}$. Again by Asplund averaging technique by lemma $3.4 X$ has an equivalent WLUR norm $|\cdot|$ such that its dual norm is LUR norm. (In particular, $|\cdot|$ is Frechet differentiable). Finally applying lemma 3.4, and Asplund averaging technique the theorem 3.1 is proved.

Theorem 3.5. [19] A Banach space $X$ is WCD if and only if there is a sequence $\left\{A_{n}\right\}$ of weak $^{*}$-compact subsets of $X^{* *}$ such that for each norm compact set $K \subseteq X$, there is an $\left\{n_{m}\right\} \subseteq \mathbb{N}$ with $K \subseteq \bigcap_{m=1}^{\infty} A_{n_{m}} \subseteq X$. In this case we say that $\left\{A_{n}\right\}$ weakly determines $X$.

A space $X$ is said to be WCD if and only if $X$ in its weak topology determined in $X^{* *}$ in its weak* ${ }^{*}$ topology.

Proof. $\Leftarrow$ Clear

$\Rightarrow$ Suppose $\left\{C_{n}\right\}$ is a sequence of weak*-compact subsets of $X^{* *}$ that weakly determines $X$. For each $i, j \in N$, let $F_{i, j}=C_{j}+\frac{1}{i} B^{* *}$. Now let $\left\{A_{n}\right\}$ be an enumeration of the finite unions of the $F_{i, j}$ and note that each $A_{n}$ is weak*compact. 
Suppose $K$ is a norm compact subset of $X$. Choose a sequence $\left\{n_{m}\right\} \subseteq N$ so that $i \in\left\{n_{m}\right\}$ if and only if $K \subseteq A_{i}$. We certainly have $K \subseteq \bigcap_{m=1}^{\infty} A_{n_{m}}$, so we need only show that $\bigcap_{m=1}^{\infty} A_{n_{m}} \subseteq X$. Let $x^{* *} \in X^{* *} \backslash X$. For each $x \in K$ there is an $l(x) \in N$ such that and $x \in C_{l(x)}$ and $x^{* *} \notin C_{l(x)}$. There is also a $j(x) \in N$ such that $x^{* *} \notin C_{l(x)}+\frac{1}{j} B^{* *}$. Note that this last set has nonempy norm inerior, so, in fact, we may find $x_{1}, x_{2}, \cdots, x_{k} \in K$ such that $K \subseteq \bigcup\left(C_{l\left(x_{t}\right)}+\frac{1}{j\left(x_{t}\right)} B^{* *}\right)$ and $x^{* *} \notin \bigcup\left(C_{l\left(x_{t}\right)}+\frac{1}{j\left(x_{t}\right)} B^{* *}\right)$, where $t=1,2, \cdots, k$. The set on the right is one of the $A_{n}$ containing $K$, hence it is one of the $A_{n_{m}}$. So we have $x^{* *} \notin \bigcap_{m=1}^{\infty} A_{n_{m}}$.

Theorem 3.6. [6] Let $X$ be a Vasak space. Then $X$ admits LUR norm.

Theorem 3.7. [6] Let $X^{*}$ be dual of a Vasak Space $X$. Then $X$ admits an equivalent LUR norm.

Question. Does every dual Vasak space admit an equivalent dual LUR norm? ([3], Cht. VII)

Proof. ([16], p. 103, 1, p. 290) The identity map $I:\left(X^{*}, w^{*}\right) \rightarrow\left(X^{*},\|\cdot\|\right)$ is slicely continuous and $X$ is norming subspace of $X^{* *}$, so $X^{*}$ admits an equivalent $w^{*}$-lower semi continuous LUR norm. Since we know that an equivalent norm on $X^{*}$ is dual if and only if it is $w^{*}$-lsc. So the norm LUR is dual. In case of WCG space, if $X$ be a (dual ) WCG space, then it admits an equivalent (dual) LUR norm [6].

Lemma 3.8. [9] Every Vasak space has an equivalent norm the dual of which is strictly convex.

Theorem 3.9. [17] Let $X$ be a Vasak Banach space. Then $X^{*}$ admits an equivalent strictly convex norm.

Proof. Let $T: X^{*} \rightarrow C_{1}\left(\sum^{\prime} \times\{0,1\}^{\alpha}\right)$ be the bounded linear one-to-one operator of (see theorem 4.1(c) [15]) Since the space $C_{1}\left(\Sigma^{\prime} \times\{0,1\}^{\alpha}\right)$ is strictly convexifiable (see Theorem $4.5[17]$ ) and $T$ is one-to-one, the space $X^{*}$ must be strictly convexifiable according to a Theorem of V. Klee ([15], Theorem 1, p. 100). This theorem is valid for WCG space as the following theorem.

Theorem 3.10. Let $X W C G$ Banach space, then there exists a set $\Gamma$ and a continuous, linear, one to-one operator $T: X^{*} \rightarrow C_{0}(\Gamma)$ which is weak-star to weak continuous ([17], p. 147). 
Theorem 3.11. [9] For a Banach space X. TFAE

(1) $X$ is a Vasak space.

(2) There exist a total set $\Gamma \subseteq B(X)$ and subset $\Gamma_{n} \subseteq \Gamma, n \in \mathbb{N}$, with the property for any $\varepsilon>0, x^{*} \in X^{*}, \gamma \in \Gamma$, there exists $n \in N, \gamma \in \Gamma_{n}$ and $\sharp\left\{\gamma^{\prime} \in \Gamma_{n}: x^{*}\left(\gamma^{\prime}\right)>\varepsilon\right\}<\aleph_{0}$. Moreover, for $\Gamma$ we can take any total subset of $B(X)$ which countably supports $X^{*}$.

Theorem 3.12. Every $W C G$ space is $W C D([8], p .384,585)$.

Proof. Let $K$ be weakly compact (and absolutely convex ; convex symmetric and bounded) set generating $X$ such that $\bigcup\{n K: n \geq 1\}$ is dense in $X, X=$ $\left.\overline{\bigcup_{n=1} n K}\right)$. we note that $X$ in its weak topology canonically embeds into $X^{* *}$ in its weak star topology. For $m, n \in \mathbb{N}$, put

$$
K_{m, n}=n K+\frac{1}{m} B\left(x^{* *}\right)=n K+\left\{X^{* *}:\|x\| \leq 1\right\},
$$

then $K_{m, n}$ is weak* compact and given $x \in X$ and $y \in X^{* *} \backslash X$, choose $m \in \mathbb{N}$ with $\operatorname{dist}(y, X)>\frac{1}{m}$ and $n$ such that $\operatorname{dist}(x, n K)<\frac{1}{m}$. Thus $x \in K_{m, n}$ and $y \notin X+\frac{1}{m}\left\{x \in X^{* *}:\|x\| \leq 1\right\} \supseteq K_{m, n}$. Thus by definition of Vasak space $X$ is a Vasak space, since $K_{m, n}$ is weak compact set in $X^{* *}$.

Theorem 3.13. A space with UG norm is a Vasak space([15], [11]).

Proof. By smulyan duality lemma " $\left\|f_{n}-g_{n}\right\| \rightarrow 0$ in the weak star topology whenever $f_{n}, g_{n} \in S\left(X^{*}\right)$ are such that $\left\|f_{n}+g_{n}\right\| \rightarrow 2$ ". For $\varepsilon>0, n \in \mathbb{N}$, put

$$
B_{n}^{\varepsilon}=\left\{x \in B_{X}:|(f-g)|<\varepsilon \text { if } f, g \in B_{X^{*}} \text { satisfy }\|f+g\|>2-\frac{1}{n}\right\},
$$

then for every $\varepsilon>0$, we get the decomposition $\bigcup_{n=1}^{\infty} B_{n}^{\varepsilon}$. we have to show that for every $\varepsilon>0$ and each $n, B_{n}^{\varepsilon} \subseteq X+4 \varepsilon B\left(X^{* *}\right)$.

If not, take $x_{0} \in \bar{B}_{n}^{\omega^{*}} \subseteq X^{* *}$ with the distance greater than $2 \varepsilon$ from $X$. Then take $F \in S\left(X^{* * *}\right)$ such that $F$ equals to zero on $X$ and $F\left(x_{0}\right)=2 \varepsilon$. Let $f_{\alpha} \in S\left(X^{*}\right)$ be such that $f_{\alpha}$ in the $w^{*}$-topology of $X^{* * *}$. Then $\left\|f_{\alpha}+f_{\beta}\right\| \rightarrow 2$ and thus $\left|\left(f_{\alpha}-f_{\beta}\right)(x)\right|<\varepsilon$ for all $x \in B_{n}^{\varepsilon}$ and for large $\alpha, \beta$. As $f_{\alpha} w^{*}$ converge to $F$, we have $\left|\left(f_{\alpha}-F\right)(x)\right| \leq \varepsilon$ for all $x \in B_{n}^{\varepsilon}$ and for large $\alpha$. Since $F=0$ on $X$, in particular on $B_{n}^{\varepsilon}$, we have $\left|f_{\beta}(x)\right| \leq \varepsilon$ for all $x \in B_{n}^{\varepsilon}$ and thus $\mid f_{\alpha}\left(x_{0}\right) \leq \varepsilon$ for large $\alpha$, from the continuity of $f_{\alpha}$ in the weak star topology of $X^{* * *}$. Since $f_{\alpha} \rightarrow F$ in the weak star topology of $X^{* * *}$. We get $\left|F\left(x_{0}\right) \leq \varepsilon\right|$ which is a contradiction. 
Remarks: 1. Separable space $\subseteq W C G \subseteq W C D$. An equivalent norm on $X$ is weakly lsc.

2. If $X$ and $X^{*}$ both are WCD, then $X$ is WCG.

3. All WCD spaces applies to $C_{0}(X)$ for locally metrizable space $X$.

Corollary 3.14. ([17], [12]) There is no bounded linear one-to-one operator $T: C_{1}(\Sigma) \rightarrow C_{0}(\Gamma)$, for any set $\Gamma$.

Theorem 3.15. [17] Every WCD Banach space $X$ admits a locally uniformly convex and smooth norm, whose dual norm on $X^{*}$ is strictly convex.

Corollary 3.16. [17] Let $X$ be a WCD Banach space. Then $X$ is a weak Asplund space.

Lemma 3.17. Let $X$ be WCD space. Then there are absolutely convex $w^{*}$-compacts $\left\{A_{i}: i \in \omega\right\}$ in $X^{* *}$ with non void norm interiors which determine $X$ in $X^{* *}$.

Theorem 3.18. Let $X$ be a WCD Banach space and $\left\{A_{i}: i \in \omega\right\}$ be the $w^{*}$-compacts from lemma 3.17. Then $X$ has a PRI subordinated to $\left\{A_{i}: i \in \omega\right\}$.

Lemma 3.19. Let $E$ and $F$ are subspaces of $X$ and $X^{*}$ respectively, let dens $. E \leq \aleph, w^{*}$-dens $. F \leq \aleph$. where $\aleph$ is a given cardinal number. Then there is a linear continuous operator $T: X^{* *} \rightarrow X^{* *}, T\left(A_{i}\right) \subseteq A_{i}$ for any $i \in \omega$ and dens $. P(X) \leq \aleph$.

Corollary 3.20. [18] Let $X$ be a WCD Banach space. Then $X$ has $w^{*}$-cardinality property, i.e. for any subspace $Y$ in $X w^{*}$-dens . $Y^{*}=$ dens.$Y$.

Proof. Assume that $X$ is WCD Banach space. As WCD Banach space has hereditary property on a closed subspace, it suffices to show that dens. $X=$ $w^{*}$-dens. $X^{*}$. Now in lemma 3.19 of above we set $E=\{0\}, F=X^{*}, \aleph=w^{*}$ dens . $X^{*}$. We can find a projection $P: X \rightarrow X$ such that $X^{*} \subseteq P^{*}\left(X^{*}\right)$, dens $. P(X) \leq \aleph$. But $P^{*}$ is identity on $X^{*}$, so $P$ is identity on $X$. This gives: dens $. X=$ dens $. P(X) \leq w^{*}$-dens.$X^{*}$. The other inequility holds for every Banach spaces. Thus dens. $X=w^{*}$-dens. $X^{*}$ implies dens. $Y=w^{*}$-dens. $Y^{*}$.

Remarks: ([18], [15]) 1. An equivalent norm on $X$ is weakly lsc.

2. If $X$ is WCD space then the following are equivalent

(1) $X$ has an equivalent Frechet differentiable norm at every point $x \in$ $X \backslash\{0\}$.

(2) $X$ has an equivalent norm the dual norm of which LUR.

4. Let $X$ be a WCD Banach space, $Y$ be a Banach space, $f$ be a continuous function from $X$ onto $Y$, then $Y$ is WCD . 
5. Let $X$ be a Banach space $X^{*}$ be WCD space, then $X$ has cardinality property. i. e. for all subspace $Y$ of $X$ dens. $Y=$ dens. $Y^{*}$.

6. All WCD spaces applies to $C_{0}(X)$ for locally metrizable space $X$.

7. A Banach space $X$ is WCD if and only if $C\left(B X^{*}\right)$ is WCD, where $B\left(X^{*}\right)$ is considered in its weak star topology. ([15], ref. therein 325]).

8. If $X$ is WCD, then it has PRI and an M-basis ([15], ref. therein 325).

9. Let $X$ be a WCD space, then $X$ has $w^{*}$ cardinality property. i.e. for any $Y \subseteq X, w^{*}$-dens $. Y^{*}=\operatorname{dens} . Y$.

10. Cardinality property of $X$ is equivalent with RNP of $X$ so every WCD dual space has RNP.

Theorem 3.21. ([12], p. 624) Let $X$ be a Banach space. If $X$ admits Gateaux differentiable norm, then $X$ is WCD.

Proof. Assume that $X$ admits Gateaux smooth norm then by Smulian's theorem

For any $f_{n}, g_{n} \in S\left(X^{*}\right),\left\|\frac{f_{n}-g_{n}}{2}\right\| \rightarrow 0$ implies $f_{n}-g_{n}$

weak $^{*}$ converges to 0 as $n \rightarrow \infty$.

For $\varepsilon>0, n \in \mathbb{N}$, put

$B_{N}^{\varepsilon}=\left\{x \in B(X):|(f-g)(x)|<\varepsilon\right.$, if $f, g \in B\left(X^{*}\right)$ satisfy $\left.\|f+g\|>2-\frac{1}{n}\right\}$.

We have $\varepsilon>0, \bigcup_{n=1}^{\infty} B_{n}^{\varepsilon} \subseteq B(X)$. We claim that for any $\varepsilon>0$ and any $n,{\overline{B_{n}^{\varepsilon}}}^{w^{*}} \subseteq$ $X+\varepsilon B\left(X^{*}\right)$. If it does not hold then there exists $x_{0}^{* *} \in \overline{B_{n}^{\varepsilon}} w^{*} \subseteq X^{* *}$ with distance greater than $2 \varepsilon$. Take $F \in S\left(X^{* * *}\right)$ such that $F$ equals to zero on $X$ and $F\left(x_{0}^{* *}\right)>2 \varepsilon$. Let $f_{\alpha} \in S\left(X^{*}\right)$ be such that $f_{\alpha} \rightarrow F$ in the $w^{*}$-topology of $X^{* *}$. Then $\left\|f_{\alpha}+f_{\beta}\right\| \rightarrow 2$ and thus $\left|\left(f_{\alpha}-f_{\beta}\right)(x)\right|<\varepsilon$ for any $x \in B_{n}^{\varepsilon}$ for large $\alpha, \beta$. As $f_{\beta}$ is weak star convergent to $F$, we have $\left|\left(f_{\alpha}-F\right)(x)\right| \leq \varepsilon$ for any $x \in B_{n}^{\varepsilon}$ and large $\alpha$. Since $F=0$ on $X$ in particular on $B_{n}^{\varepsilon}$, we have $\left|f_{\alpha}(x)\right| \leq \varepsilon$ for any $x \in B_{n}^{\varepsilon}$. and then $\left|f_{\alpha}\left(x_{0}^{*}\right)\right| \leq \varepsilon$ for large $\alpha$. From the continuity of $f_{\alpha}$ in $\left(X^{* *}, w^{*}\right) \cdot f_{\alpha} \rightarrow F$ in $\left(X^{* *}, w^{*}\right)$, we get $\left|F\left(x_{0}^{* *}\right)\right| \leq \varepsilon$ which is a contradiction. The existence of countable family $\left\{\bar{B}_{n}^{\frac{1}{m}} w^{*}: m, n \in \mathbb{N}\right\}$ ensures that $X$ is WCD space. 
Remark. [10] Clearly, every weakly $\sigma$-shrinking total bounded set in a Banach space $X$ makes $X$ have property $G$. Thus it follows from Proposition 2 that Vasak spaces have property $G$. We conjecture that the non Vasak space $C(\Omega)$ constructed in [AM, p. 421] (see also [F, Sec. 7.3]) has property $G$.

Remark 1. If $Y$ is closed subspace of the WCD space $X$, to show that $Y$ is WCD it suffices to consider the sets $\left(K_{n} \bigcap Y^{\perp \perp}\right)$, where $Y^{\perp \perp} \subseteq X^{* *}$.

Corollary 3.22. ([15]) Every Vasak space has an equivalent norm the dual of which is strictly convex.

Corollary 3.23. [18] Let $X$ be a WCD Banach space. Then $X$ has $w^{*}$ cardinality property, i. e. for every subspace $Y$ in $X w^{*}$-dens $. Y^{*}=$ dens . .

Proof. Since WCD property is hereditary on closed subspaces, it suffices to show that $w^{*}$-dens $. X^{*}=$ dens.$X$. By the step $\mathrm{v}$ of theorem 1 in [13], we set $E=\{0\}, F=X^{*}, \aleph=w^{*}$-dens.$X^{*}$. So we can find a projection $P: X \rightarrow X$ such that $X^{*} \subseteq P^{*}\left(X^{*}\right)$, dens $. P(X) \leq \aleph$. But then $P^{*}$ is identity on $X$. This immediately gives: dens $. X=$ dens $. P(X) \leq w^{*}-$ dens.$X^{*}$. The other inequility holds in every Banach spaces.

Corollary 3.24. [18] Let $X$ be a WCD Banach space. Then:

(1) There is a set $\Gamma$ and a linear continuous one-to-one operator $L$ from $X$ into $c_{0}(\Gamma)$;

(2) X has LUR norm.

Proof. Both results holds in case of WCG space. These are proved in [9] and [10] respectively. But those proofs work in every Banach space, subspaces of which have PRI (all subspaces or even only all complemented subspace) ( see Theorem 3.1, reference thereof [13]) shows that WCD spaces are that type.

Theorem 3.25. Let $X$ be WCD Banach space. TFAE:

(1) Every $Y \subseteq X$ has $P R I\left\{P_{\alpha}: \omega \leq \alpha \leq \mu\right\}$ such that $\left\{P_{\alpha}^{*}: \omega \leq \alpha \leq \mu\right\}$ is PRI of $Y^{*}$.

(2) $X$ has an equivalent Frechet differentiable norm away from zero.

(3) $X$ has equivalent norm the dual norm of which is LUR.

(4) Every subspace $Y \subseteq X$ has shrinking Markusevic basis, i. e. a biorthogonal pair $\left(H_{1}, H_{2}\right), H_{1} \subseteq Y, H_{2} \subseteq Y^{*}$ such that $\overline{s p} H_{1}=Y$ and $\overline{s p} H_{2}=Y^{*}$.

Theorem 3.26. [18] (1) Let $X$ be WCD space, $Y$ a Banach space, and $f$ is linear continuous mapping of $X$ onto $Y$. Then $Y$ is $W C D$.

(2) Let $X$ be a Banach space such that $X^{*}$ is $W C D$. Then $X$ has cardinality property, i.e. dens $Y=$ dens.$Y^{*}$ for any subspace of $Y$ of $X$. 
Theorem 3.27. [18] If $X$ and $X^{*}$ be both WCD Banach spaces, then $X$ is $W C G$.

Theorem 3.28. [2] Let $(X,\|\cdot\|)$ be a WCD Asplund space. Then $X$ admits an equivalent Frechet differentiable norm whose dual norm is locally uniformly rotund. Moreover $\mathrm{X}$ is weakly compactly generated.

Lemma 3.29. [11] Assume that $M$ is a bounded weakly closed set in a Banach space $X$. Assume that the norm $\|\cdot\|$ of $X$ is dually $M-2 R$. Then $M$ is weakly compact.

Proof. Let $S \subseteq M$ be a countable subset of $M$ and assume that $x \in$ $\bar{S}^{w^{*}} \subseteq X^{* *}$ be such that $x \notin X$. Let $F \in S\left(X^{* * *}\right)$ be such that $F \in X^{\perp}$ and $F(x)=\operatorname{dist}(x, X)>0$. Let $\left\{y_{i}\right\} \subseteq B\left(X^{* * *}\right)$ be $\operatorname{such}$ that $\sup _{i} F\left(y_{i}\right)=$ 1. Let $f_{n} \in S\left(X^{*}\right)$ be such that $\lim _{n \rightarrow \infty}\left(f_{n}-F\right)(s)=0$ for all $s \in S$, that $\lim _{n \rightarrow \infty}\left(f_{n}-F\right)\left(y_{i}\right)=0$ for all $i$ and that $\lim _{n \rightarrow \infty}\left(f_{n}-F\right)(x)=0$.

The existence of $\left\{f_{n}\right\}$ follows from a metrizable version of the Goldstine theorem ([8], p. 73). Then $\lim _{m, n \rightarrow \infty}\left\|f_{m}+f_{n}\right\|=2$ and thus by the rotundity assumed, $\lim _{n \rightarrow \infty}\left(f_{n}-F\right)(s)=0$ and $\lim _{n \rightarrow \infty}\left(f_{n}-F\right)(x)=0$ uniformly on $S \bigcup\{x\}$. As all $f_{n}$ are continuous on $S \bigcup\{x\} \subseteq X^{* *}$ in its relative point wise topology, so is their uniform limit on this set, which is not the case as $F$ is zero on $S$ and $F(x)>0$. Therefore $x \in X$ and thus $M$ is countably weakly compact which means that $M$ is weakly compact by the Eberlein-Smulyan theorem (cf. e.g. [8], Ch. IV).

Theorem 3.30. [11] Assume that $X$ is separable Banach space $X$ and $M$ is a bounded set in $X$. Then $M$ weaklly relatively compact if and only if $X$ admits an equivalent dually $M-2 R$.

Theorem 3.31. [11] A Banach space $X$ is $W C G$ if and only if $X$ admits a dually $M-2 R$ norm for some bounded total set in $X$.

Proof. Assume that $X$ is WCG. Then there is a bounded linear one-toone operator $T$ from $X^{*}$ into some $c_{0}(\Gamma)$. Let $\|\cdot\|_{D}$ be the Day norm on $c_{0}(\Gamma)$. By the result of Hajek and Johanis $([14])$, the norm defined on $X^{*}$ by $\|f\|_{1}^{2}+\|T f\|_{D}^{2}$ is dually M-2R, where $M=T^{*}\left(e^{\alpha}\right),\left\{e^{\alpha}\right\}$ are the unit vectors in $c_{0}(\Gamma)$. By Lemma 3.29 , the set $M$ is relatively weakly compact in $X$ and the closed linear hull of it equals to $X$. The other implication is contained in Lemma 3.29. 


\section{Conclusion}

From above observations we derive the special case of the above open question which has positive answer. But general case still remains open.

\section{Acknowledgements}

The first author would like to express his gratitude to his Ph. D advisor, Prof. Prakash Muni Bajracharya, for his encouragement, constant support, helpful remarks and insightful comments on this paper. I also would like to express my deep gratitude to UGC of Nepal for financial support. The work of third author was supported by the National Natural Science Foundation of China (11571378).

\section{References}

[1] A. Amanollah, H. Haghshenas, Some classical and recent results concerning renorming theory. Thai J. Math., 10 (2012), no. 2, 481-495.

[2] A. Aviles, Weakly countably determined spaces of high complexity. Studia Math., 185 (2008), no. 3, 291-303. http://dx.doi.org/10.4064/sm185-3-6

[3] R. Deville, G. Godefroy, V. Zizler, Smoothness and renormings in Banach spaces. Pitman Monographs and Surveys in Pure and Applied Mathematics, 64. Longman Scientific and Technical, Harlow; copublished in the United States with John Wiley and Sons, Inc., New York, 1993.

[4] J. Diestel, Geometry of Banach spacesselected topics. Lecture Notes in Mathematics, Vol. 485. Springer-Verlag, Berlin-New York, http://dx.doi.org/10.1007/BFb0082079 1975.

[5] M. Fabian, Each weakly countably determined Asplund space admits a Frechet differentiable norm. Bull. Austral. Math. Soc., 36 (1987), no. 3, 367-374. http://dx.doi.org/10.1017/S000497270000366X

[6] M. Fabian, On a dual locally uniformly rotund norm on a dual Vasak space. Studia Math. 101 (1991), no. 1, 69-81.

[7] M. Fabian, Gateaux differentiability of convex functions and topology. Weak Asplund spaces. Canadian Mathematical Society Series of Monographs and Advanced Texts. A Wiley-Interscience Publication. John Wiley and Sons, Inc., New York, 1997.

[8] M. Fabian, P. Habala,P. Hájek, V. S. Montesinos, J. Pelant, V. Zizler, Functional analysis and Infinite-dimensional geometry, Springer-Verlag, New York, 2001. http://dx.doi.org/10.1007/978-1-4757-3480-5

[9] M. Fabian, G. Godefroy, V. Montesinos,and V. Zizle, Inner characterizations of weakly compactly generated Banach spaces and their relatives. J. Math. Anal. Appl., 297 (2004), no. 2, 419-455. http://dx.doi.org/10.1016/j.jmaa.2004.02.015 
[10] M. Fabian, V. Montesinos, V. Zizler, The Day norm and Gruenhage compacta. Bull. Austral. Math. Soc., 69 (2004), no. 3, 451-456. http://dx.doi.org/10.1017/S0004972700036236

[11] M. Fabian, V. Montesinos and V. Zizler, Smoothness in Banach spaces. Selected problems. RACSAM. Rev. R. Acad. Cienc. Exactas Fís. Nat. Ser. A Mat., 100 (2006), no. 1-2, 101125.

[12] M. Fabian, P. Habala, P. Hájek, V. Montesinos, V. Zizler, Banach space theory. The basis for linear and nonlinear analysis. CMS Books in Mathematics/Ouvrages de Mathématiques de la SMC. Springer, New York, 2011. http://dx.doi.org/10.1007/978-14419-7515-7

[13] J. R. Giles, D. A. Gregory, and Brailey Sims, Characterisation of normed linear spaces with Mazur's intersection property. Bull. Austral. Math. Soc., 18 (1978), no. 1, 105-123. http://dx.doi.org/10.1017/S0004972700007863

[14] P. Hajek, M. Johanis, Characterization of reflexivity by equivalent renorming. J. Funct. Anal., 211 (2004), no. 1, 163-172. http://dx.doi.org/10.1016/S0022-1236(03)00264-7

[15] W. B. Johnson and J. Lindenstrauss. Handbook of the geometry of Banach spaces. Vol. 2. Edited by North-Holland, Amsterdam, 2003. unit 18, p 784-830.

[16] A. Malto, J. Orihuela, S. Troyanski, M. Valdivia, A nonlinear transfer technique for renorming. Lecture Notes in Mathematics, 1951. Springer-Verlag, Berlin, 2009.

[17] S. Mercourakis, Sophocles, On weakly countably determined Banach spaces. Trans. Amer. Math. Soc., 300 (1987), no. 1, 307-327.

[18] L. Vasak, On one generalization of weakly compactly generated Banach spaces. Studia Math., 70 (1981), no. 1, 11-19.

[19] D. Wilkins, The strong WCD property for Banach spaces. Internat. J. Math. Math. Sci., 18 (1995), no. 1, 67-70. 